\title{
(6) OPEN ACCESS \\ Predictive factors for non-response to intravitreal ranibizumab treatment in age-related macular degeneration
}

\author{
Misa Suzuki, Norihiro Nagai, Kanako Izumi-Nagai, Hajime Shinoda, Takashi Koto, \\ Atsuro Uchida, Hiroshi Mochimaru, Kenya Yuki, Mariko Sasaki, Kazuo Tsubota, \\ Yoko Ozawa
}

Additional material is published online only. To view please visit the journal online (http://dx.doi.org/10.1136/ bjophthalmol-2013-304670).

Department of Ophthalmology Keio University School of Medicine, Tokyo, Japan

\section{Correspondence to} Yoko Ozawa, Laboratory of Retinal Cell Biology, Department of Ophthalmology, Keio University School of Medicine, 35 Shinanomachi, Shinjuku-ku, Tokyo 160-8582, Japan; ozawa@a5.keio.jp

Received 21 November 2013 Revised 24 February 2014 Accepted 20 March 2014 Published Online First

7 April 2014

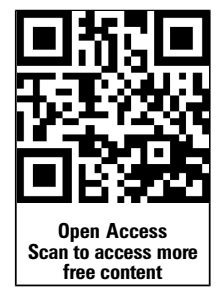

\begin{abstract}
Background/aims To study the initial characteristics and response to intravitreal ranibizumab (IVR) treatment of age-related macular degeneration (AMD).

Methods We reviewed the clinical records of 141 eyes in 141 AMD patients who received monthly IVR for 3 months and thereafter pro re nata (PRN) injections for 9 months as the first treatment for AMD. Patients whose best corrected visual acuity (BCVA) worsened at month 12 , and those with increased exudative fundus findings after IVR or an increased central retinal thickness of more than $100 \mu \mathrm{m}$ at month 12 , were considered to be non-responders as judged by BCVA and fundus findings, respectively. Non-responders' initial characteristics were analysed using logistic regression models.

Results $14.9 \%$ of eyes were non-responders as judged by BCVA, and $17.0 \%$ were non-responders as judged by fundus findings. Initial fibrovascular pigment epithelial detachment (PED) (OR 22.9, 95\% Cl 2.61 to 201) and serous PED (OR 4.12, 95\% Cl 1.08 to 15.8) were associated with non-response as judged by BCVA. Initial fibrovascular PED (OR 33.5, 95\% CI 2.95 to 381 ) and type 1 choroidal neovascularization (OR 6.46, 95\% Cl 1.39 to 30.0 ) were associated with non-response, as judged by fundus findings.
\end{abstract}

Conclusions Although most AMD responded to IVR, non-responders had initial clinical characteristics that might be informative for managing their treatment.

\section{INTRODUCTION}

Anti-vascular endothelial growth factor (anti-VEGF) therapy, which was first developed as a cancer treatment, ${ }^{1}$ is now used worldwide as the main therapy for treating choroidal neovascularisation (CNV) in age-related macular degeneration (AMD), a chronic condition..$^{2-4}$ One of the anti-VEGF drugs, ranibizumab (Lucentis; Genentech, San Francisco, California, USA) is a recombinant humanised monoclonal antibody fragment that inhibits human VEGF, and is injected several times intravitreally to suppress the proliferation and hyperpermeability of CNV. The MARINA and ANCHOR studies ${ }^{3}{ }^{4}$ of intravitreal ranibizumab (IVR) therapy showed that the average best corrected visual acuity (BCVA) of AMD patients increased after monthly ranibizumab treatment, while the average BCVA after photodynamic therapy (PDT) decreased, ${ }^{4}$ launching a new era in the field of AMD treatment.

Thus, the efficacy of IVR for the majority of patients has been demonstrated. ${ }^{3}{ }^{4}$ However,
anti-VEGF therapy is not completely effective for every patient and some AMD patients still lose their visual acuity after treatment. Furthermore, a previous report showed that $45 \%$ of patients treated with bevacizumab, another anti-VEGF drug that is used off-label as a treatment for AMD,${ }^{5}$ are non-responders, and that efficacy depends on the initial lesion size and reading ability, although this report was based on 6 months of follow-up and only BCVA was used for judging non-responders. Another group reported that $15 \%$ of an IVR group were non-responders as judged by fundus findings at month 3 , and vitreo-retinal adherence was the only ophthalmic factor influencing prognosis. ${ }^{6}$ Thus, AMD cases unresponsive to an anti-VEGF drug may not be unusual. Since other therapies, including other anti-VEGF drugs with different drug designs, and PDT, are available, it would be helpful for AMD patients if the efficacy of IVR could be determined as early as possible in their treatment so that non-responders could begin other, more appropriate therapy.

In this study, we compared the initial characteristics of 141 responder and non-responder eyes of 141 patients who received pro re nata (PRN) IVR after monthly IVR for the first 3 months as the first treatment for AMD. The non-responders were identified 12 months after the first IVR either by BCVA or by fundus findings, including optical coherence tomography (OCT) images, with the two sets of findings analysed separately. We sought to define predictive factors for non-responsiveness to IVR as such information would be valuable for the optimal management of AMD.

\section{MATERIALS AND METHODS}

The study followed the tenets of the Declaration of Helsinki and was approved by the ethics committee at Keio University School of Medicine.

\section{Study participants}

This was a retrospective study based on detailed medical chart review. The study included 141 eyes of 141 patients with visual loss due to neovascular AMD and treated with IVR at the Medical Retina Division Clinic (AMD Clinic) of the Department of Ophthalmology, Keio University Hospital (Tokyo, Japan) between March 2009 and May 2012. All patients were initially treatment naive and had attended our clinic for at least 12 months, during which the only treatment they received was IVR. 
Patients who had received any previous treatment for AMD before initial IVR were excluded.

\section{Eye examinations}

All subjects underwent BCVA measurement with the refraction test throughout the course of treatment, slit-lamp examination, and binocular indirect ophthalmoscopy after pupil dilation with $0.5 \%$ tropicamide.

\section{Fluorescein and indocyanine green angiography}

Fluorescein angiography and indocyanine green (ICG) angiography were performed using a Topcon TRC 50DX retinal camera (Topcon, Tokyo, Japan) to diagnose AMD and determine its subtypes, polypoidal choroidal vasculopathy (PCV) and retinal angiomatous proliferation (RAP). Angiographic grading was carried out according to the TAP criteria by retina specialists in our clinic (MS, NN and YO). ${ }^{7}$ Using fluorescein angiography, significant pigment epithelial detachment (PED) was defined according to the lesion diameter: serous PED $>4$ disc diameters (DD), haemorrhagic PED $>4$ DD, and fibrovascular PED $>3$ DD.

\section{OCT}

OCT was used to evaluate central retinal thickness (CRT), intraretinal oedema, subretinal fluid accumulation and PED. CRT was defined as the distance between the internal limiting membrane and the presumed retinal pigment epithelium (RPE) at the fovea. OCT was performed at every follow-up visit using an OCT-C7 (Nidek, Gamagori, Japan) or Heidelberg Spectralis OCT (Heidelberg Engineering, Dossenheim, Germany) instrument. Measurement was performed by referring to the scale bars in the OCT system.

\section{Treatment with ranibizumab and follow-up}

Ranibizumab $(0.5 \mathrm{mg}, 0.05 \mathrm{~mL})$ was injected intravitreally under sterile conditions via the pars plana once a month for 3 months, as an induction phase. Re-injections were given if the OCT image showed evidence of any fluid in the macula, identified as macular oedema or subretinal fluid, or enlargement of a PED at the time of follow-up examinations. The follow-up was carried out every month, but when fluid or haemorrhage was absent for more than 2 months, the interval was extended up to 2 months. Any new subretinal or intraretinal haemorrhage or unexplained visual loss of more than 0.2 at $\log$ MAR score was also treated. At each follow-up visit, BCVA was measured and other eye examinations, including OCT recordings, were carried out. The average number of injections was 4.6 2.2 (including the initial three injections and additional PRN injections).

\section{Determination of non-responders by BCVA}

Initial BCVA (before the initial IVR) and BCVA at the 12-month visit were compared. Non-responders were identified as those whose BCVA had worsened by more than 0.2 in the logMAR score.

\section{Determination of non-responders by fundus findings including OCT}

Initial fundus findings were recorded at each visit. Non-responders were identified as those in whom exudative fundus findings (PED, subretinal fluid, macular oedema, haemorrhage) had increased or had appeared after treatment, or in whom the CRT increased by more than $100 \mu \mathrm{m}$ between the time of initial IVR and month 12.

\section{Statistical analysis}

Commercially available software (SPSS, V.18.0) was used for the statistical analysis. The demographic characteristics of the responders and non-responders were compared using the Mann-Whitney U test, and statistical significance was defined as $\mathrm{p}<0.05$. Adjusted ORs and 95\% CIs for non-responders were estimated with logistic regression models to examine the effects of the confounding factors on the unadjusted results. In the multivariable analysis, potential risk factors for non-responders such as type $1 \mathrm{CNV}$, serous PED, haemorrhagic PED or fibrovascular PED were adjusted for age, gender, CRT and CNV size at the time of initial IVR.

\section{RESULTS}

Based on BCVA, 21 eyes of 21 patients among a total of 141 eyes (14.9\%) were non-responders at 12 months, while 24 eyes of 24 patients among 141 eyes (17.0\%) were non-responders at 12 months based on fundus findings (table 1). The nonresponders, as judged by BCVA, were significantly older on average, initially, than the responders, by the Mann-Whitney U test. There were no differences in gender, initial BCVA, AMD types, initial CRT or initial greatest linear dimension (GLD). In contrast, the non-responders as judged by fundus findings included more type $1 \mathrm{CNV}$.

The initial characteristics of the fundus findings are shown in table 1 . Non-responders as judged by fundus findings included more cases with initial fibrovascular PED and fewer cases with macular oedema than responders.

We next analysed the correlation between initial fundus findings and the non-responders (table 2). Fibrovascular PED (OR 22.9, 95\% CI 2.61 to 201) and serous PED (OR 4.12, 95\% CI 1.08 to 15.8 ) were significantly correlated with non-response as judged by BCVA, and fibrovascular PED (OR 33.5, 95\% CI 2.95 to 381 ) and type $1 \mathrm{CNV}$ (OR 6.46, 95\% CI 1.39 to 30.0 ) were significantly correlated with non-response as judged by fundus findings, both after multivariable adjustment. Other findings shown in table 2, as well as serous retinal detachment, macular oedema and subretinal haemorrhage, did not correlate with non-response in either analysis. One non-responding case, as judged by fundus findings, with initial fibrovascular PED is shown in figure 1.

Although non-responders were not correlated with typical AMD or PCV groups overall, details were further analysed. The distribution of non-responders according to AMD subtype, as judged by fundus findings, is shown in figure 2 .

Importantly, there was only one non-responder with predominantly classic CNV (6.3\%) and no non-responder with minimally classic type among the typical AMD cases. However, among PCV cases, five eyes with multiple polyps (26.3\%) were nonresponders, while no eye with a single polyp was a non-responder.

Interestingly, there were no non-responders as judged by fundus findings among the RAP cases (stage 1, five eyes; stage 2, six eyes; stage 3, four eyes), although two eyes (13.3\%) of nonresponders as judged by BCVA developed an RPE tear after the fifth injection, and also had fibrovascular PED initially. A case of RAP in which the BCVA improved is shown in figure 3.

\section{DISCUSSION}

At 12 months after the initial IVR, $14.9 \%$ of eyes were nonresponders as determined by BCVA, and $17.0 \%$ were nonresponders as determined by fundus findings. The average age 
Table 1 Demographics and initial ocular characteristics

\begin{tabular}{|c|c|c|c|c|}
\hline & $\begin{array}{l}\text { BCVA responders } \\
\text { (120 eyes) }\end{array}$ & $\begin{array}{l}\text { BCVA non-responders } \\
\text { ( } 21 \text { eyes) }\end{array}$ & $\begin{array}{l}\text { Fundus responders } \\
\text { (117 eyes) }\end{array}$ & $\begin{array}{l}\text { Fundus non-responders } \\
\text { ( } 24 \text { eyes) }\end{array}$ \\
\hline Age, mean \pm SD & $72.6 \pm 10.1$ & $75.9 \pm 5.4^{*}$ & $72.9 \pm 9.9$ & $73.6 \pm 7.9$ \\
\hline Male/female gender, no. eyes (male \%) & $80 / 40(66.6)$ & 15/6 (71.4) & $78 / 39(66.6)$ & $17 / 7(70.8)$ \\
\hline BCVA, logMAR (mean $\pm S D)$ & $0.39 \pm 0.44$ & $0.52 \pm 0.35$ & $0.41 \pm 0.45$ & $0.41 \pm 0.33$ \\
\hline \multicolumn{5}{|l|}{ AMD type } \\
\hline Typical AMD & 73 & 10 & 64 & 19 \\
\hline PCV & 34 & 9 & 38 & 5 \\
\hline RAP & 13 & 2 & 15 & 0 \\
\hline $\mathrm{CRT}$, mean $\pm \mathrm{SD}(\mu \mathrm{m})$ & $404.8 \pm 185.7$ & $484.2 \pm 269.5$ & $426.2 \pm 209.2$ & $361.8 \pm 141.0$ \\
\hline $\mathrm{GLD}$, mean $\pm \mathrm{SD}(\mu \mathrm{m})$ & $3432.1 \pm 2207.4$ & $4007.8 \pm 2104.2$ & $3429.8 \pm 2085.1$ & $3947.0 \pm 2675.9$ \\
\hline Type 1 CNV, no. eyes (\%) & $74(61.7)$ & $18(75.0)$ & $68(58.1)$ & $22(91.7)^{\star *}$ \\
\hline \multicolumn{5}{|l|}{ Fundus findings, no. eyes (\%) } \\
\hline Serous PED & $50(41.7)$ & $11(52.4)$ & $52(44.4)$ & $9(37.5)$ \\
\hline Fibrovascular PED & $35(29.2)$ & $11(52.4)$ & $30(25.7)$ & $16(66.6)^{* *}$ \\
\hline Haemorrhagic PED & $11(9.2)$ & $6(28.6)$ & $14(20.0)$ & $3(4.2)$ \\
\hline Serous retinal detachment & $71(59.2)$ & $13(61.9)$ & $70(59.8)$ & $14(54.2)$ \\
\hline Macular oedema & $30(33.3)$ & $6(28.6)$ & $33(28.2)$ & $3(12.5)^{* *}$ \\
\hline Retinal haemorrhage & $38(32.5)$ & $7(33.3)$ & $39(33.3)$ & $6(25.0)$ \\
\hline
\end{tabular}

at the time of initial IVR of the non-responders as judged by BCVA was greater than that of responders.

Fibrovascular PED and serous PED were correlated with nonresponders as judged by BCVA, and fibrovascular PED and type $1 \mathrm{CNV}$ were correlated with non-responders as judged by fundus findings.

Although IVR has generally improved the prognosis of AMD dramatically, ${ }^{3} 4$ some individuals did not achieve effects. Yamashiro et $\mathrm{al}^{8}$ reported that $14.3 \%$ of PCV cases and $14.3 \%$ of typical AMD cases discontinued IVR therapy and started PDT due to the lack of IVR effect, and Kruger Falk et al ${ }^{9}$ showed that $15 \%$ of neovascular AMD cases are bad or nonresponders. In this study, the ratio of non-responders or untreatable cases was quite similar to previous data, although the criteria for the non-responders were different. As regards the number of injections in the first 12 months, our result showed fewer injections than the CATT $^{10}$ or IVAN studies. ${ }^{11}$ However, the number of injections was similar to previous reports showing an effect of IVR monotherapy at year $1,,^{12}$ and the report by Kruger Falk et $a l^{9}(8.7$ injections in the mean follow-up duration of 23.3 months including a monthly injection for 3 months in the loading phase), suggesting that nonresponse was not due to insufficient treatment. The larger number of IVR treatments in the PRN group of the CATT study may, at least in part, be due to differences in the inclusion criteria: the CATT study only includes eyes with BCVA of between 20/25 and 20/320 initially, while our study included those who had better BCVA and may have included milder cases reflecting the recent trend of rapid care with early detection.

Interestingly, fibrovascular PED was a risk factor for the nonresponders as judged by both BCVA and fundus findings. This may be explained by physiology: the fibrous tissue beneath the RPE may have decreased oxygen diffusion from the choriocapillaris, ${ }^{13}$ resulting in interruption of active transport of exudative fluid across the RPE. ${ }^{14}$ This parameter was also correlated with non-response as judged by BCVA, most likely because the ability of the RPE to maintain the microenvironment would also be reduced and the photoreceptor function may have been impaired.

Initial serous PED was observed in 61 of 141 eyes, 50 of which $(41.7 \%)$ were non-responders as judged by BCVA. Interestingly, detailed analysis showed that serous PED with multiple polyps did not respond to IVR in all cases. A previous report showed that an absence of serous PED or of haemorrhagic PED was correlated with decreased exudative fluid following IVR. ${ }^{3}{ }^{4}$ Our findings are consistent with this report in terms of the negative association of serous PED with a good prognosis after IVR.

In contrast, surprisingly, all the cases with RAP had serous PED, and all of them responded to IVR with a reduction or elimination of fluid. Although the effect of combined therapy with PDT and an anti-VEGF drug for RAP has been previously shown, ${ }^{15}$ the efficacy of IVR monotherapy has also been recently reported, ${ }^{16}$ consistent with our data. Moreover, official guidelines for AMD treatment developed in Japan ('Treatment Guidelines for Age-related Macular Degeneration', http://www. nichigan.or.jp/member/guideline/aging_macular_degeneration. pdf) and the UK ('Age-related Macular Degeneration Guidelines for Management' of the Royal College of Ophthalmologists, http://www.rcophth.ac.uk/page.asp?section=451) include antiVEGF monotherapy for RAP treatment. Further study to determine the pathogenesis of RAP and compare the effects of the treatments may be important in the future as there may be differences in the underlying mechanisms of serous PED in typical AMD, PCV and RAP.

The previously reported rate of PCV eye resistance to IVR was $14.3 \%,{ }^{8}$ which was similar to our data (five eyes of 43 cases, 11.6\%). However, the fundus findings after IVR in the PCV cases was not uniform in this study. There were no nonresponders among eyes with a single polyp, while five eyes (26.3\%) among those with multiple polyps were nonresponders. Whether the polyp was single or multiple may have led to the different results as the amount of abnormal vascularisation might have contributed to this difference. 
Table 2 Predictors of non-responders after intravitreal ranibizumab therapy

\begin{tabular}{|c|c|c|c|c|c|c|c|c|c|c|c|c|}
\hline \multirow[b]{3}{*}{ As judged by } & \multicolumn{6}{|l|}{ BCVA } & \multicolumn{6}{|c|}{ Fundus finding } \\
\hline & \multicolumn{3}{|l|}{ Crude } & \multicolumn{3}{|c|}{ Multivariate, adjusted } & \multicolumn{3}{|l|}{ Crude } & \multicolumn{3}{|c|}{ Multivariate, adjusted } \\
\hline & $p$ Value & OR & $95 \% \mathrm{Cl}$ & $p$ Value & OR & $95 \% \mathrm{Cl}$ & $p$ Value & OR & $95 \% \mathrm{Cl}$ & $p$ Value & OR & $95 \% \mathrm{Cl}$ \\
\hline Age & 0.143 & 1.041 & 0.997 to 0.097 & - & - & - & 0.767 & 1.007 & 0.961 to 1.055 & - & - & - \\
\hline Gender & 0.668 & 0.800 & 0.288 to 2.219 & - & & - & 0.692 & 0.824 & 0.315 to 2.152 & - & - & - \\
\hline BCVA & 0.189 & 1.953 & 0.719 to 5.307 & 0.551 & 0.732 & 0.262 to 2.044 & 0.984 & 0.990 & 0.352 to 2.783 & 0.940 & 0.956 & 0.995 to 1.001 \\
\hline \multicolumn{13}{|l|}{ AMD type } \\
\hline PCV† & 0.191 & 1.932 & 0.719 to 5.191 & 0.154 & 2.125 & 0.755 to 5.983 & 0.134 & 0.443 & 0.153 to 1.284 & 0.083 & 0.374 & 0.123 to 1.138 \\
\hline RAPt & 0.889 & 1.123 & 0.220 to 5.725 & 0.722 & 0.726 & 0.125 to 4.234 & $-\ddagger$ & $-\ddagger$ & $-\ddagger$ & $-\ddagger$ & $-\ddagger$ & $-\ddagger$ \\
\hline CRT & 0.093 & 1.002 & 1.000 to 1.004 & - & - & - & 0.154 & 0.998 & 0.995 to 1.001 & - & - & - \\
\hline GLD & 0.273 & 1.000 & 1.000 to 1.000 & - & - & - & 0.297 & 1.000 & 1.000 to 1.000 & - & - & - \\
\hline Type 1 CNV & 0.191 & 2.378 & 0.648 to 8.723 & 0.141 & 2.741 & 0.717 to 10.479 & $0.026^{*}$ & 5.500 & 1.221 to 24.771 & $0.017^{*}$ & 6.462 & 1.390 to 30.036 \\
\hline \multicolumn{13}{|l|}{ Fundus finding } \\
\hline Serous PED & $0.007^{* *}$ & 5.937 & 1.623 to 21.722 & $0.039^{*}$ & 4.123 & 1.077 to 15.782 & 0.355 & 1.946 & 0.477 to 7.946 & 0.335 & 2.156 & 0.453 to 10.266 \\
\hline Haemorrhagic PED & $0.043^{*}$ & 12.526 & 1.082 to 144.986 & 0.096 & 8.443 & 0.685 to 104.061 & $-\ddagger$ & $-\ddagger$ & $-\ddagger$ & $-\ddagger$ & $-\ddagger$ & $-\ddagger$ \\
\hline Fibrovascular PED & $0.004^{* *}$ & 13.882 & 2.360 to 81.656 & $0.005^{* *}$ & 22.896 & 2.613 to 200.607 & $0.002^{* *}$ & 30.526 & 3.379 to 275.817 & $0.005^{* *}$ & 33.507 & 2.949 to 380.691 \\
\hline
\end{tabular}

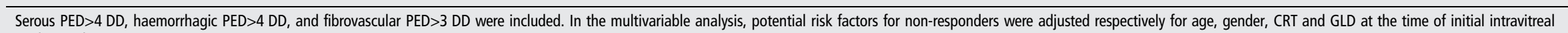
ranibizumab.

${ }^{*} \mathrm{p}<0.05,{ }^{* *} \mathrm{p}<0.01$.

Reference typical AMD.

Unanalysable because all subjects were responders.

AMD, age-related macular degeneration; BCVA, best corrected visual acuity; CNV, choroidal neovascularisation; CRT, central retinal thickness; GLD, greatest linear dimension; PCV, polypoidal choroidal vasculopathy; PED, pigment epithelial detachment; RAP, retinal angiomatous proliferation. 


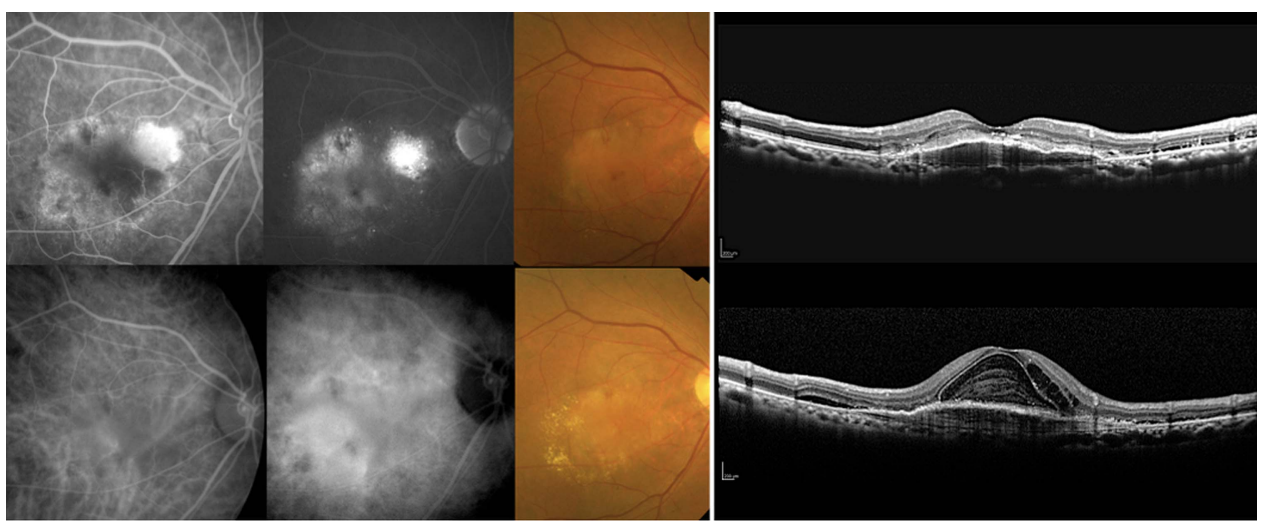

Figure 1 A non-responder as judged by fundus findings with fibrovascular pigment epithelial detachment (PED). This was a 71-year-old man with typical age-related macular degeneration and best corrected visual acuity (BCVA) of 0.3 (logMAR 0.52) at the time of initial intravitreal ranibizumab (IVR). Fluorescein (top row, left two panels, early phase and late phase) and indocyanine green (bottom row, left two panels, early phase and late phase) angiograms were consistent with the findings of the fundus colour photograph and an optical coherence tomography image showing fibrovascular PED before initial IVR (top row, right two panels). After seven IVR injections, the BCVA worsened to 0.15 (logMAR 0.82) at month 12. Although the fibrovascular PED did not change markedly, there was an increase in the subretinal and intraretinal fluid at month 12 (bottom row, right two panels).

Type $1 \mathrm{CNV}$ also correlated with non-responders as judged by fundus findings, which may, at least partly, have been because RPE barrier function was preserved in these cases and drug penetration was insufficient. Initial type $1 \mathrm{CNV}$ was not a risk factor for non-response as judged by BCVA; this might be related to the remaining RPE barrier function which would inhibit rapid progression of the AMD lesion.

We recommend that for those with characteristics of IVR nonresponse, such as serous or fibrovascular PED or type $1 \mathrm{CNV}$ before initial IVR, the efficacy of IVR is estimated to be lower than the results of the clinical studies, which only report average efficacies, ${ }^{17}$ in evaluating the benefit of treatment. Patients with non-responders' characteristics may choose other anti-VEGF drugs, or PDT at an earlier time point, which may improve prognosis since they can avoid inefficient and unnecessary treatment.

Nine of 141 eyes (6.4\%) were non-responders as judged by both BCVA and fundus findings (see online supplementary table S1), and fibrovascular PED was the only predictor for nonresponse (see online supplementary table S2). Those nonresponders as judged by BCVA and those as judged by fundus findings did not necessarily overlap, which may be an interesting finding for future study. The limitations of this study were the rather small size of the population and the different AMD types in the population. However, we hope our results will encourage further studies to establish the criteria for non-responders in order to avoid inefficient treatment and achieve benefits for patients.

\section{cases}

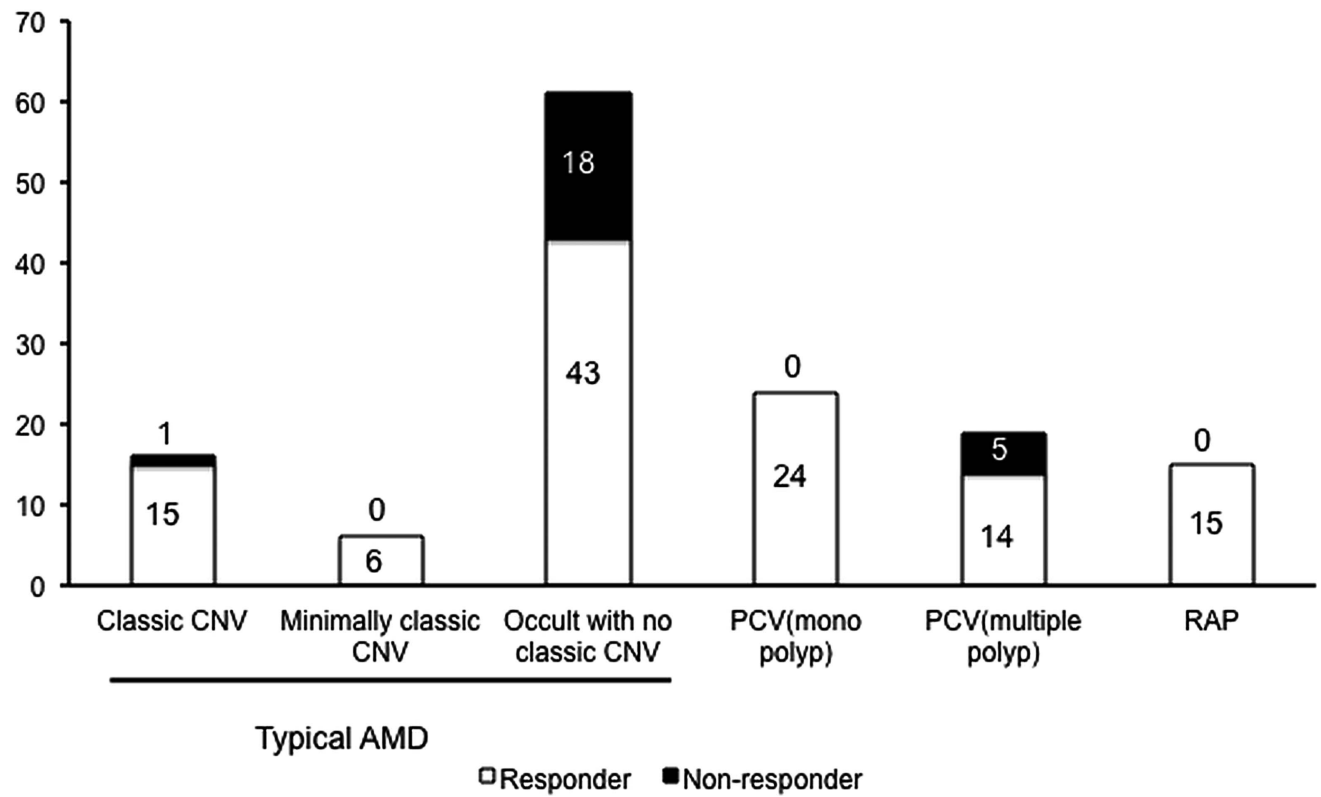

Figure 2 Distribution of non-responders according to AMD type, as judged by fundus findings. Non-responders are shown in black in each bar. The numbers of non-responders and responders are included in the graph. AMD, age-related macular degeneration; CNV, choroidal neovascularisation; PCV, polypoidal choroidal vasculopathy; RAP, retinal angiomatous proliferation. 

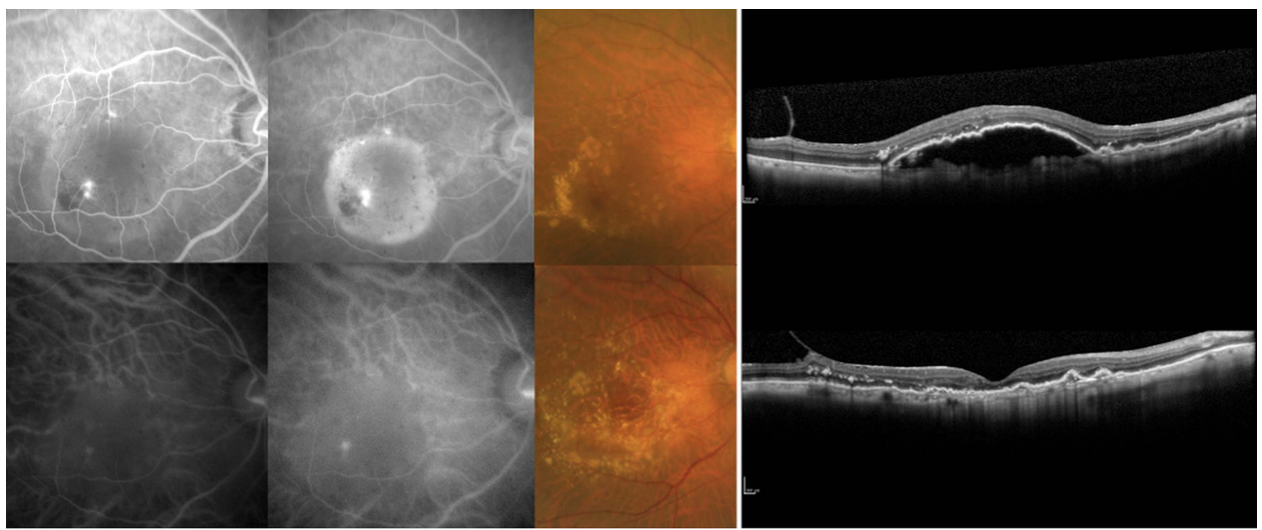

Figure 3 A responder as judged by fundus findings with retinal angiomatous proliferation (RAP). This was an 85 -year-old man with an initial best corrected visual acuity (BCVA) of 0.1 (logMAR 1.0). Initial fluorescein (top row, left two panels, early phase and late phase) and indocyanine green (bottom row, left two panels, early phase and late phase) angiograms and colour fundus photograph (top row, centre panel) showed a typical RAP lesion. Initial central retinal thickness (CRT) measured in the optical coherence tomography image was $708 \mu \mathrm{m}$ (top row, right panel). At month 12 , the BCVA was improved to 0.4 (logMAR 0.4), all the exudative fundus findings had improved or disappeared, and the CRT was reduced to $194 \mu \mathrm{m}$ (bottom row, right two panels).

Acknowledgements We thank the orthoptists and medical staff of our clinic for technical assistance.

\section{Competing interests None.}

Patient consent Obtained.

Provenance and peer review Not commissioned; externally peer reviewed.

Open Access This is an Open Access article distributed in accordance with the Creative Commons Attribution Non Commercial (CC BY-NC 3.0) license, which permits others to distribute, remix, adapt, build upon this work non-commercially, and license their derivative works on different terms, provided the original work is properly cited and the use is non-commercial. See: http://creativecommons.org/ licenses/by-nc/3.0/

\section{REFERENCES}

1 Ferrara N. Vascular endothelial growth factor as a target for anticancer therapy. Oncologist 2004;9(Suppl 1):2-10.

2 Gragoudas ES, Adamis AP, Cunningham ET, et al. Pegaptanib for neovascular age-related macular degeneration. N Engl J Med 2004;351:2805-16.

3 Rosenfeld PJ, Brown DM, Heier JS, et al. Ranibizumab for neovascular age-related macular degeneration. N Engl J Med 2006;355:1419-31.

4 Brown DM, Kaiser PK, Michels M, et al. Ranibizumab versus verteporfin for neovascular age-related macular degeneration. N Engl J Med 2006;355:1432-44.

5 Lux A, Llacer H, Heussen FM, et al. Non-responders to bevacizumab (Avastin) therapy of choroidal neovascular lesions. Br J Ophthalmol 2007:91:1318-22.

6 Krebs I, Glittenberg C, Ansari-Shahrezaei S, et al. Non-responders to treatment with antagonists of vascular endothelial growth factor in age-related macular degeneration. Br J Ophthalmol 2013;97:1443-6.
7 Photodynamic therapy of subfoveal choroidal neovascularization in age-related macular degeneration with verteporfin: one-year results of 2 randomized clinical trials-TAP report. Treatment of age-related macular degeneration with photodynamic therapy (TAP) Study Group. Arch Ophthalmol 1999;117:1329-45.

8 Yamashiro K, Tomita K, Tsujikawa A, et al. Factors associated with the response of age-related macular degeneration to intravitreal ranibizumab treatment. $\mathrm{Am} J$ Ophthalmol 2012;154:125-36.

9 Kruger Falk M, Kemp H, Sorensen TL. Four-year treatment results of neovascular age-related macular degeneration with ranibizumab and causes for discontinuation of treatment. Am J Ophthalmol 2013;155:89-95.e3.

10 Martin DF, Maguire MG, Ying GS, et al. Ranibizumab and bevacizumab for neovascular age-related macular degeneration. N Engl J Med 2011;364:1897-908.

11 Chakravarthy U, Harding SP, Rogers CA, et al. Ranibizumab versus bevacizumab to treat neovascular age-related macular degeneration: one-year findings from the IVAN randomized trial. Ophthalmology 2012;119:1399-411.

12 Hikichi T, Higuchi M, Matsushita T, et al. One-year results of three monthly ranibizumab injections and as-needed reinjections for polypoidal choroidal vasculopathy in Japanese patients. Am J Ophthalmol 2012;154:117-24.e1.

13 Friedlander M. Fibrosis and diseases of the eye. J Clin Invest 2007:117:576-86.

14 Marmor MF. Control of subretinal fluid: experimental and clinical studies. Eye (Lond) 1990;4(Pt 2):340-4.

15 Saito M, lida T, Kano M. Combined intravitreal ranibizumab and photodynamic therapy for retinal angiomatous proliferation. Am J Ophthalmol 2012;153:504-14.e1.

16 Gharbiya M, Allievi F, Recupero V, et al. Intravitreal bevacizumab as primary treatment for retinal angiomatous proliferation: twelve-month results. Retina 2009:29:740-9.

17 Ying GS, Huang J, Maguire MG, et al. Baseline predictors for one-year visual outcomes with ranibizumab or bevacizumab for neovascular age-related macular degeneration. Ophthalmology 2013;120:122-9. 